Journal of Engineering and Applied Sciences 15 (2): 643-652, 2020

ISSN: 1816-949X

(C) Medwell Journals, 2020

\title{
Resource Allocation in OFDMA Femtocell Based LTE and 5G Networks with QoS Guarantees
}

\author{
Amenah A. Hafez, Yasser M. Jaamour and Khaldoun I. Khorzom \\ Department of Telecommunication, Higher Institute for Applied Sciences and Technology, \\ Damascus, Syria
}

\begin{abstract}
Expanding network capacity and guaranteeing the Quality of Service (QoS) are significant goals in fifth-Generation (5G) for high densities of mobile terminals. Femtocell-based 5G is an essential radio access technology that meets the exponentially increasing demand. Femtocells have emerged as an efficient solution for improving the capacity and coverage of wireless cellular networks, especially, for indoor wireless users. However because of the limited wireless radio resources, resource allocation is a key issue in femtocell networks. Motivated by this challenge in this study, we propose an efficient resource allocation approach that satisfies the QoS requirements for High-Priority (HP) users while serving Best-Effort (BE) users effectively as possible. The user differentiation strategy ensures the QoS guarantee uponthe priority level of each user. We consider major metrics for performance evaluation which are: the rate of rejected users, throughput satisfaction rate, spectrum spatial reuse and fairness. Dedicated simulations prove that our proposal outperforms one of the most effective techniques in the literature.
\end{abstract}

Key words: LTE, 5G, resource allocation, femtocell, OFDMA, QoS, clustering

\section{INTRODUCTION}

In recent years, wireless networks have been suffering from exponentially increasing demand to support various new applications and data services such as online games, Voice over IP (VoIP), Internet of Things (IoT), Vehicle-to-everything (V2X) communications, e-Health, Mobile Edge Computing (MEC), video streaming and cloud computing which require high-speed network access. Explosive demands for such applications and services increase internet traffics by 50 60\% each year (Lee and Lee, 2015). It is estimated by IMT-2020 (5G) promotion group that the global mobile data traffic will grow by more than 200 times from 2010-2020 and by nearly 20,000 times from 2010-2030. 5G is fast emerging to address the challenges caused by an exponential increase in wireless data traffic.

According to recent research, most of the data traffic today takes place in indoor environments (Ghaith et al., 2016). However, cellular users in indoor environments may have difficulty in receiving high data rate services from a Macro Base Station (MBS) due to the non-LOS propagation losses. Furthermore, macrocell coverage becomes costly to serve indoor consumers with large service demands. Therefore, one of the potential solutions for enhancing indoor coverage and capacity is to deploy indoor Femtocell Access Points (FAPs). The femtocell technology also called home base station which installed by users is the best way to support a higher data rate and provides a satisfactory QoS requirements for its users in a short range of coverage (Zhang et al., 2014; Pourkabirian et al., 2018). Femtocell communication is a cost-effective technology that requires low power, operates in a licensed spectrum and connects to the cellular network via. broadband backhaul connection such as optical fiber or Digital Subscriber Line (DSL) (Mohammadi et al., 2018; Vaezpour et al., 2017). Both of users and operators profit from femtocells. Users enjoy high-quality links due to the closeness between transmitter and receiver. On the other hand, operators decrease the Operational Expenditure (OPEX) and Capital Expenditure (CAPEX) due to the traffic offloading and user's self-deployment of FAPs (Zhang et al., 2014). Despite these benefits, the deployments of femtocells suffer from several technical challenges such as co-tier interference (interference between FAPs) and cross-tier interference (interference between macrocells and FAPs). Due to the interference effect on network performance, choosing a proper access strategy is required.

There are mainly three strategies for femtocell access: closed, open and hybrid access.In closed access, only subscribed users can connect with FAPs. However, FAPs users will suffer from cross-layer interference if both macro and FAP users work on the same frequency band. On the other hand, open access allows all wireless users to access the FAPs with no constraints. One advantage of this method is that it prevents cross-layer interference between macro and FAPS users. As a disadvantage, open

Corresponding Author: Amenah A. Hafez, Department of Telecommunication,

Higher Institute for Applied Sciences and Technology, Damascus, Syria 
access will increase the number of signaling and handoffs. Furthermore, FAPs users may not achieve the required data rate. Hybrid access allows the connectivity of nonsubscribers while restricting the number of resources that can be shared. It allows all macro users to connect with FAPs and guarantees the desirable performance and rate for FAP users. Moreover, it can reduce cross-tier interference and improve FAP user's performance reliability. Hence, this access mechanism, adopted in this study, enhances network capacity (Mohammadi et al., 2018; Hatoum et al., 2013; Lopez-Perez et al., 2009).

There are mainly two schemes to implement: a two-tier cellular network, namely, split-spectrum and shared spectrum schemes. In the first scheme, the spectrum is divided into two independent parts, one used by FAPs and the other used by macrocells. Although, the cross-tier interference can be mitigated with an orthogonal channel assignment, this scheme has poor spectral efficiency. In the second scheme, FAPs and macrocells share the total allocated spectrum. This scheme has better spectral efficiency. However, it leads to cross-tier interference that needs to be addressed (Lee and Lee, 2015; Hatoum et al., 2013). In practice, the femtocell is deployed over the existing macrocell network by spectrum sharing rather than spectrum splitting between tiers due to the scarce availability of spectrum and absence of coordination between macrocells and femtocells on spectrum allocation. As a result of spectrum sharing, it is essential to deal with cross-tier interference in femtocell networks (Zhang et al., 2014; Li et al., 2010; Lee et al., 2010).

Motivated by the above-mentioned challenges, we propose an efficient resource allocation approach that satisfies QoS requirements for HP users while serving BE users effectively as possible. Our contribution focuses on the downlink femtocell network where the multiuser access technology used is the Orthogonal Frequency Division Multiple Access OFDMA (adopted by the 3GPP LTE/LTE-Advanced and 5G systems) (TS 38.211, NR, 2019; ETSI TS 136.300 V10.5.0, 2011).

Literature review: Recently, resource allocation and interference mitigation issues in OFDMA femtocell networks have drawn significant attention from the researchers. They seek to exploit resources efficiently to achieve the overall system objectives while mitigating cross-layer interference and co-layer interference. Different methods are performed to realize these objects. Some of relevant works arecomprehensively presented in the following.

Game theoretical resource allocation approaches in femtocell networks are proposed by Pourkabirian et al. (2018), Mohammadi et al. (2018). Researchers by Pourkabirian et al. (2018) proposed a game-theoretic approach for two-tier femtocell networks that takes into consideration macro user's activity and user's QoS requirements in resource allocation. They formulated the problem as the Vickrey-Clarke-Groves (VCG) auction game theory to model the competitive behavior of users for resources. It is shown that the approach maximized social welfare and satisfied user's QoS requirement. However, VCG mechanism suffers from low revenue of individual users (Day and Milgrom, 2008). Whereas by Mohammadi et al. (2018), the researchers introduced an approach for sharing spectrum in a Cognitive Radio (CR) system with Femto Users (FUs) and Macro Users (MUs) as primary and secondary users, respectively. The problem is formulated as Stackelberg-game joined with a convex optimization problem.

By Zhang et al. (2014), researchers focused on sub-channel and power allocation problems in OFDMA based two-tier femtocell networks in which a central macrocell is overlaid with spectrum-sharing femtocells and taking into account heterogeneous femto user's QoS requirements. They formulated the problem into a non-convex mixed integer-programming problem. Then they transformed it into a convex problem by relaxing the combinatorial sub-channel allocation constraint into a continuous variable and then solved it. However, its computational complexity was high, so, they proposed a low-complexity algorithm that can provide satisfactory performance in both uplink and downlink.

The proposed scheme by Lee and Lee (2015) categorizes the entire time-frequency resource blocks of the overlaid cellular network into dedicated and shared one and allocates these resources based on user location and user-required data rate to expand the user accommodation capacity. They performed cross-tier handovers from the macrocell to the femtocell to maximize the total packet throughput.

By $\mathrm{Li}$ et al. (2012), researchers studied resource allocation in open access OFDMA femtocell networks to guarantee QoS of the neighboring macrocell user MU in dead zone and limit cross-tier interference to other MUs. They used the idea of CR technology in femtocell networks and proposed a resource allocation method. Under such a cognitive femtocell framework, the researchers formulated a joint sub-channel and power optimization problem and introduced an algorithm using dual decomposition methods. It has shown that the proposed algorithm for the open-access scheme could achieve substantial transmission rate gains over the closed access scheme.

Two centralized approaches are presented by Lopez-Perez et al. (2009), called Centralized-Dynamic Frequency Planning C-DFP and orthogonal assignment algorithm. In the former approach, a sub-channel broker receives requirements and interference information from the femtocells and/or the macrocells, so as to compute the best resource allocation, the tradeoff is between 
computational complexity and optimality. This scheme can easily converge to the optimum. However, it is more appropriate for small-sized femtocell networks. The latter one divided the spectrum into two independent sets, one is used by the macrocells and the other is used by femtocells, so as to maximize the satisfaction of the required QoS. However, this scheme does not consider the co-layer interference and not suitable for high-density networks.

By Li and Sousa (2012), an autonomous scheduling scenario is provided using a co-channel cognitive approach. Each FAP listens to the surrounding and identifies the suitable Resource Block (RB) to be allocated.

A collaborative approach for the cross-layer interference is proposed by Sahin et al. (2009). The FAPs work with the MBS to control the unreliable information because of the spectrum sensing lacks.

The researchers by Sundaresan and Rangarajan (2009) proposed a distributed resource allocation algorithm, called Distributed Random Access (DRA). The resources, time-frequency slots (chunks) are divided between femtocells and macrocells based on the gradient ascent/descent heuristic. The aim is to allocate these chunks to macro and femto users such that total system utility is maximized. It is shown that this algorithm is fully distributed with an acceptable worst-case performance guarantee. However, this approach cannot guarantee QoS in a realistic scenario because of its pseudo-random nature and it is more appropriate for medium-size networks.

By Lee et al. (2010), the researchers presented a Fractional Frequency Reuse (FFR) technique that sets the frequency reuse factor to alleviate co-layer interference. In this case, femtocells are arranged depending on the amount of reciprocal interference by a centralized femtocell gateway that defines the minimum number of orthogonal sub-channels for each group and adjusts the transmit power of each femtocell based on the received signal strength. The researchers by Liang et al. (2012) proposed a greedy algorithm for physical resource block allocation with QoS classes for different services. The suggested method assumes the use of a central controller and gateway organizing network access for femto installations and responsible for resource allocation. However, it is appropriate only for small-sized femtocell networks.

A trade-off between the distributed and the centralized methods is a clustering approach. Cluster elements share information with each other which enhances scheduling reliability while reducing network complexity, since, it alleviates the loading through the backhaul (Jin et al., 2011), hence, we consider in this paper such an approach.

By Bouras and Diles (2015) researchers propose an algorithm that determines the resource allocation in femtocell clusters based on hybrid access policy. They categorize three classes of users that can be admitted by a femtocell. They explore particularly how femtocells may best utilize their available resources in order to increase their provided capacity when neighboring femtocells are present.

Another cluster-based hybrid scheme proposed by Hatoum et al. (2013) called QoS-based femtocell Resource Allocation (QFCRA) which consists of three main phases such as femtocell cluster formation, intra-cluster resource allocation and inter-cluster resource contention resolution. It aims to satisfy a maximum number of QoS-constrained HP users and simultaneously serve the BE users effectively as possible.

Accordingly, we aim in this study to optimize the exploitation of the available resources. We distribute the available radio resource blocks to the users in order to mitigate the interference as well as responding to the user throughput demands. Moreover, we underline the QoS impact by differentiating between two users types: HP and BE users.

\section{MATERIALS AND METHODS}

Problem formulation: In this study, we first define both the network model used in our analysis and the propagation model. Then we formulate the joint resource allocation and admission control as multi-objective optimization problem.

Network model: In our study, we focus on the downlink communications based on OFDMA whose frame structure can be viewed as time-frequency RBs. We call them chunks. We consider an OFDMA (e.g., LTE/5G-based) femtocells network consisting of several FAPs representing residential or home networks. We adopt the shared spectrum approach as by Hatoum et al. (2013), Lopez-Perez et al. (2009), Sundaresan and Rangarajan, (2009) and Chandrasekhar and Andrews (2009). We assume an orthogonal channel assignment to eliminate the cross-layer interference between femtocells and the macrocell. A chunkis the smallest unit of resource that can be assigned to a user. One 5G New Radio (NR) chunk contains either 14 (normal Cyclic Prefix (CP)) or 12 (Extended CP) OFDM symbols in time domain and 12 sub-carriers in frequency domain similar to LTE. In LTE a chunk bandwidth is fixed to $180 \mathrm{kHz}$ but in NR it is not fixed and depends on sub-carrier spacing (3GPP specs) (TS 38.211, NR, 2019).

Scheduling is done on a slot basis. In LTE, each sub-frame consists of two equally sized slots of $0.5 \mathrm{msec}$. Whereas in 5G there are various numerologies. Hence, sub-frame contains different number of slots depending on sub-carrier spacing.Each sub-frame consists of $2^{\mu}$ slots and slot length $=1 \mathrm{msec} / 2^{\mu}$ as shown in Table 1 (3GPP specs) (TS 38.211, NR, 2019). 
J. Eng. Applied Sci., 15 (2): 643-652, 2020

Table 1: 5G NR subcarrier spacing for normal cyclic prefix

\begin{tabular}{lccccc}
\hline$\mu$ & $\Delta \mathrm{f}=2^{\mu} .15[\mathrm{kHz}]$ & $\mathrm{N}_{\text {symbol }}^{\text {slot }}$ & Duration of $\mathrm{N}_{\text {symbol }}^{\text {slot }}(\mathrm{msec})$ & $\mathrm{N}_{\text {slot }}^{\text {frame }}$ & \\
\hline 0 & 15 & 14 & 1.0000 & 10 & $\mathrm{~N}_{\text {sslot }}^{\text {subfame }}$ \\
1 & 30 & 14 & 0.5000 & 20 & 1 \\
2 & 60 & 14 & 0.2500 & 40 & 2 \\
3 & 120 & 14 & 0.1250 & 80 & 8 \\
4 & 240 & 14 & 0.0625 & 160 & 16 \\
\hline
\end{tabular}

A certain number of users attach to each FAP. User demands represent the required bandwidth, expressed in number of required chunks. The relation between required chunks of user $u$, denoted by $\mathrm{D}_{\mathrm{u}}$ and the through put requirement, denoted by $\mathrm{TP}_{\mathrm{u}}{ }^{\text {req }}$ can be written as follows (Hatoum et al., 2013):

$$
\mathrm{D}_{\mathrm{u}}=\left[\frac{\mathrm{TP}_{\mathrm{u}}^{\mathrm{req}}}{\psi \cdot \mathrm{eff}}\right]
$$

Where:

$\psi \quad: \quad\left(\mathrm{SC}_{\text {ofdm }} \cdot \mathrm{SY}_{\text {ofdm }}\right) / \mathrm{T}_{\text {slot }}$ is a parameter that depends on the network configuration

$\mathrm{SC}_{\text {ofdm }} \quad$ : The numbers of sub-carriers and

and $\mathrm{SY}_{\text {ofdm }} \quad$ symbols per chunk, respectively

$\mathrm{T}_{\text {slot }} \quad:$ One slot duration in time units

eff $_{\mathrm{u}} \quad: \quad$ The efficiency (bits/symbol) of the used Modulation and Coding Scheme (MCS)

We differentiate between users according to their demand requirements thus two types of users are considered: HP users and BE users (Ghaith et al., 2016 Hatoum et al., 2013):

- HP users: their requirements need to be fully satisfied in term of a desired QoS. HP users may be the owner of the FAP

- BE users: we try to reach as maximum as possible their demands with different levels of applications. BE users may be visitors of the FAP

Since, HP users are strict with their QoS requirements and the total amount of network resources is bounded an admission control strategy for HP users is then needed. An HP user is surely admissible only when the network has sufficient resources to meet the QoS demands. In this study, for simplicity, we assume afixed transmissions power for all FAPs as by Hatoum et al. (2013), Lopez-Perez et al. (2009), Sundaresan and Rangarajan (2009), Liang et al. (2012) and Chandrasekhar and Andrews (2009).

Propagation model: The indoor FAPsare assumed to be in rooms. Two link types are considered: The Line of Sight (LOS) case where FAP and UE are in the same room and the Non-Line of Sight (NLOS) casewhere FAP and UE are in different rooms. In NLOS case, wall attenuation must be considered. Further, Floor Loss (FL) of the vertical radiations for propagation from floor to floor is modeled and the floor loss must be added to the path-loss calculated for each floor. Therefore, the A1 path loss model is considered as the propagation model for the frequency range 2-6 GHz developed in Winner. The path loss model is thus, summarized in the following form:

$$
\mathrm{PL}=\mathrm{A} \log _{10}(\mathrm{~d}[\mathrm{~m}])+\mathrm{B}+\mathrm{Clog} \log _{10}\left(\frac{\mathrm{f}_{\mathrm{c}}[\mathrm{GHz}]}{5.0}\right)+\mathrm{X}
$$

In Eq. 2, $\mathrm{d}$ is the distance between FAP and UE in [m] $f_{c}$ is the carrier frequency in $\mathrm{GHz}$, the fitting parameter $\mathrm{A}$ includes the path-loss exponent, parameter $\mathrm{B}$ is the intercept, $\mathrm{C}$ describes the path loss frequency dependence, the shadow fading distribution is log-normal. $\mathrm{X}$ is an optional, environment-specific term (e.g., wall attenuation in the NLOS scenario).

Resource allocation problem: Let $\mathrm{F}$ be the set of FAPs, $\mathrm{H}$ the set of HP users and B the set of BE users in the network. Moreover, let $\mathrm{I}_{\mathrm{f}}$ and $\mathrm{I}_{\mathrm{u}}$ be the set of interfering femtocells of the FAP F and the interfering set of user $u$, respectively. These sets are determined using the minimum required Signal to Interference plus Noise Ratio (SINR) values and the indoor path loss model. Actually, each user $\mathrm{u}$ within the FAP boundary calculates the ratio of the received signal from his FAP to the signals received from the neighboring FAPs. If this ratio is lower than the minimum required SINR threshold, this user notifies its FAP. The neighboring FAPs will then be considered as an interferer for $\mathrm{F}$ and will belong to the set $\mathrm{I}_{\mathrm{f}}$. Besides, each user within these neighboring FAPs is considered interferer of the user $u$ and should not be assigned the same chunk as $u$. Hence, the set $I_{u}$ can be written as follows: For each user $\mathrm{u}$ attached to the FAP F, $\mathrm{I}_{\mathrm{u}}=\left\{v \neq \mathrm{u} \mid v \in \mathrm{F} \cup \mathrm{I}_{\mathrm{f}}\right\}$. Users use the received SINR to calculate the Channel Quality Indicator (CQI) then they report it to the network. Based on these measurement reports, a FAP can decide to reallocate the chunk or allocate a different MCS in the case of using link adaptation. Also, we denote by $\mathrm{D}_{\mathrm{HP}}^{\mathrm{u}}$ and $\mathrm{D}_{\mathrm{BE}}^{\mathrm{v}}$ the demand of the HP user $\mathrm{u} \in \mathrm{H}$ and the $\mathrm{BE}$ user $v \in \mathrm{B}$, respectively. We also define for each HP user $\mathrm{u}$ (respectively, BE user $v$ ) the binary resource allocation vector, denoted by $\Delta_{\mathrm{HP}}^{\mathrm{u}}$, (respectively $\Delta_{\mathrm{BE}}^{v}$ ) with one or zero in position $\mathrm{j}$ according to whether the chunk $\mathrm{j}$ is used or not. 
Our objective is to find the optimal resource allocation of a set of chunks in each FAP to deliver user's data while minimizing the interference between FAPs and at the same time providing HP users QoS requirements as well as maximizing the throughput for BE users. Because the limited capacity of the network if the QoS requirements of HP users exceed the available resources, then satisfying all HP users becomes infeasible. Hence, we define the set of admitted HP users in the network, denoted by $\mathrm{H}^{*} \subseteq \mathrm{H}$, for which the QoS requirements are fully satisfied. Our first objective will be then to maximize the cardinality of the set $\mathrm{H}^{*}$. In addition, for each BE user $v$ we define a variable $G_{B E}(v)$ which represents the gap between the required and the allocated resources of $v$ that is (Hatoum et al., 2013:

$$
G_{\mathrm{BE}}(v)=\left(\frac{D_{\mathrm{BE}}^{v}-\sum_{\mathrm{j}=1}^{\mathrm{M}} \Delta_{\mathrm{BE}}^{v}(j)}{D_{\mathrm{BE}}^{v}}\right)
$$

where, $\mathrm{M}$ denotes the number of available resources (i.e., chunks) in the network. Our second objective will be then to minimize the maximum value of GB while trying to achieve as much fairness as possible and serving the maximum number of $\mathrm{BE}$ users. The joint resource allocation and admission control problem for $\mathrm{HP}$ and $\mathrm{BE}$ users can be formulated as shown in the following problem. Joint resource allocation and admission control for $\mathrm{HP}$ and $\mathrm{BE}$ users problem:

- Maximize the number of the accepted HP users, i.e., $\max \left|\mathrm{H}^{*}\right|$

- Minimize the maximum value of BE users' gaps, i.e., $\min \left[\max _{v \in \mathrm{B}} \mathrm{G}_{\mathrm{BE}}(v)\right]$

Subject to:

- The resource scheduler must guarantee that admitted HP users are fully satisfied, i.e.

$$
\forall \mathrm{u} \in \mathrm{H}^{*}: \sum_{\mathrm{j}=1}^{\mathrm{M}} \Delta_{\mathrm{HP}}^{\mathrm{u}}(\mathrm{j})=\mathrm{D}_{\mathrm{HP}}^{\mathrm{u}}
$$

- BE users should be given chunksless or equal to their demands, i.e.

$$
\forall v \in \mathrm{B}: \sum_{\mathrm{j}=1}^{\mathrm{M}} \Delta_{\mathrm{BE}}^{v}(\mathrm{j}) \leq \mathrm{D}_{\mathrm{BE}}^{v}
$$

- Two interfering users cannot be given the same chunk, i.e.

$$
\forall \mathrm{j}=1, \ldots, \mathrm{M}, \forall \mathrm{u} \in \mathrm{H}^{*} \cup \mathrm{B}, \forall v \in \mathrm{I}_{\mathrm{u}}: \Delta^{\mathrm{u}}(\mathrm{j})+\Delta^{v}(\mathrm{j}) \leq 1
$$

- The resources vector $\Delta$ is a binary vector, i.e.

$$
\forall \mathrm{j}, \forall \mathrm{u} \in \mathrm{H}^{*} \cup \mathrm{B}, \Delta^{\mathrm{u}}(\mathrm{j}) \in\{0,1\}
$$

This problem is amulti-objective optimization problem and has been proved to be NP-hard (Aissi et al., 2005). To solve it, we first subdivide it into sub problems through clustering. The corresponding problem will be then solved sequentially. That is, we will try to satisfy HP users first and then resolve for $\mathrm{BE}$ users with the remaining resources.

Problem resolution: In this study, we present the algorithm used to resolve the problem. It consists of two main stages cluster formation which is the same as QFCRA algorithm (Hatoum et al., 2013), resource allocation per cluster. We call our algorithm Enhanced QFCRA (EQFCRA). In what follows, we present these stages.

Cluster formation: In this stage, FAPs gather surrounding information. When powered on, a FAP will listen to surrounding transmissions (i.e., neighboring FAPs' control channel and reference signal transmissions) and gather information through measurements collected from users attached to it or via. a receiver function within the FAP. Based on this information, the FAP F can compute the number of interfering femtocells (i.e., $\left|\mathrm{I}_{\mathrm{f}}\right|$ called interference degree) and transmitit a long with its Physical Cell Identity (PCI) to each one of them. Therefore, each FAP will have a list containing the interference degree of neighboring femtocells and will decide whether it is a Cluster-Head $(\mathrm{CH})$ or is attached to a neighboring cluster. The $\mathrm{CH}$ election algorithm can be described as follows:

Each femtocell elects the $\mathrm{CH}$ as the one with the highest interference degree among it sone-hop neighbors. If it is not $\mathrm{CH}$ itself, the femtocell acts as a Cluster-Member (CM) of a $\mathrm{CH}$ chosen by its immediate neighbors. If more than one unique $\mathrm{CH}$ is chosen by the neighboring femtocells, the one with the highest interference degree is elected as $\mathrm{CH}$ to minimize the collision of chunks between femtocells (if equal degrees, a random tie-break is used).

If no $\mathrm{CH}$ is chosen by the neighboring femtocells (i.e., all neighbors act as CMs and are already associated to other clusters), the femtocell is attached to the cluster of the neighbor with the highest interference degree. To avoid large cluster size due to the attachment of such femtocells to neighboring clusters, we set a threshold on its size denoted by. When the threshold is reached, the corresponding femtocell will act as an isolated $\mathrm{CH}$.

Resource allocation per cluster: Once the femtocell network is partitioned into clusters, the second step is to jointly allocate resources to all FAPs within each cluster taking into account. QoS requirements of attached users, $I_{u}$ of users located near the edge of the cluster. To achieve this, each cluster-member reports to its corresponding $\mathrm{CH}$ 
the required resources to satisfy its user's demands. Then, each $\mathrm{CH}$ tries to resolve individually the original problem (the resource allocation problem for all users).

A good solution of the original problem could be attained by resolving sequentially the two objectives of the resource allocation problem. In the following, we present our approach to resolve the resource allocation problem for HP users first, then for BE users.

HP user admission control and resource allocation: As stated earlier, we need to choose a subset of HP users for which the allocation problem is feasible. Since, the objective is to maximize the number of satisfied HP users, the cardinality of such subset has to be the maximum of all such subsets. This problem is equivalent to the IIS (Irreducible Infeasible Set) problem. An IIS is an infeasible set of constraintsof which any proper subset is feasible. That is if were moveany one constraint from an IIS, the IIS will be feasible. In linear programming, this set is often difficult to determine. Hence, a useful approach was introduced by Brown and Graves called "elastic programming". It consists of adding an extra variable allowing constraints to "relax" in order to increase the feasibility region. In other terms if an HP user cannot fulfill its demands with the available resources, it will use a certain elastic variable to complete its requirements. Thus, for each HP user $u$, we introduce an elastic variable $\mathrm{e}_{\mathrm{u}}$. To locate the inconsistent constraints, (Chinneck and Dravnieks, 1991) proposed to create a new objective function-minimize the sum of elastic variables and then perform a "filtering" where the constraints having elastic variables greater than zero form the set of inconsistent constraints. Hence, the optimization problem for HP users will be formulated as follows:

\section{HP users problem:}

- Minimize the sum of HP user's elastic variables, i.e., $\min \Sigma_{\mathrm{u} \in \mathrm{H}} \omega_{\mathrm{u}} \times \mathrm{e}_{\mathrm{u}}$

Subject to:

- The sum of the allocated chunks to any HP user with their elastic variable is equal or bigger then their demand, i.e., $\forall \mathrm{u} \in \mathrm{H}: \Sigma^{\mathrm{M}}{ }_{\mathrm{j}=1} \Delta_{\mathrm{HP}}^{\mathrm{u}}(\mathrm{j})+\mathrm{e}_{\mathrm{u}} \geq \mathrm{D}_{\mathrm{HP}}^{\mathrm{u}}$

- Two interfering users cannot be give the same chunk, i.e., $\forall \mathrm{j}, \forall \mathrm{u} \in \mathrm{H}, \forall v \in \mathrm{I}_{\mathrm{u}}: \Delta^{\mathrm{u}}{ }_{\mathrm{HP}}(\mathrm{j})+\Delta^{v}{ }_{\mathrm{HP}}(\mathrm{j}) \leq 1$

- The elastic variables are positive integers, i.e., $\forall \mathrm{u} \in \mathrm{H}$ : $\mathrm{e}_{\mathrm{u}} \geq 0$

In this problem, $\omega_{\mathrm{u}} \in \Re_{+}$are weighting coefficients used to set priority levels between different HP users. When $\omega_{u}=1, \forall \mathrm{u}$ then all users are given equal priority. Note that an optimal value of anelastic variable $e^{*}$ should be zero for the corresponding HP user to be fully satisfied.
On the other hand, a non-zero solution indicates the need for more resources than available in the network to satisfy the corresponding HP user. Therefore, we will admitin to the network only those HP users whose corresponding elastic variables reaches zero. The complete resolution algorithm for HP users is described as follows:

- $\quad$ First, each CH resolves HP users problem for all HP users within the cluster

- Then, we determine the set S of HP users for which the elastic variables are greatert han zero

- $\quad$ The power set of $S$, denoted by $P(S)$ is generated. It is composed by all subsets of $\mathrm{S}$. That is assuming $\mathrm{S}$ is a finite set with cardinality $|S|=n$ and then $P(S)$ is finite and its cardinality $|\mathrm{P}(\mathrm{S})|=2^{\mathrm{n}}$

- Afterward, the algorithm remove the elements $X$ within $\mathrm{P}(\mathrm{S})$ one at a time starting with the lowest cardinality sub-sets and resolves the above problem for the remaining set $\mathrm{H}^{*}$ of $\mathrm{HP}$ users where $\mathrm{H}^{*}=\mathrm{H}-\mathrm{X}$

- If, $\sum_{\mathrm{u} \in \mathrm{H}^{*}} \omega_{\mathrm{u}} \times \mathrm{e}_{\mathrm{u}}>0$, the previously removed element is reinserted, since, its removal did not allow the feasibility of the problem and the next element within PS is removed. HP users problem is resolved again on the new set $\mathrm{H}^{*}$

- The process is stopped if $\sum_{\mathrm{u} \in \mathrm{H}^{*}} \omega_{\mathrm{u}} \times \mathrm{e}_{\mathrm{u}}>0$

At the end of this process, the set $\mathrm{H}^{*}$ of admitted HP users (for which the original problem is feasible) as well as the corresponding allocation matrix $\mathrm{A}_{\mathrm{HP}}$ of dimensions $\left|\mathrm{H}^{*}\right| \times \mathrm{M}$ are determined. The next step is now to allocate the remaining resources to BE users.

BE users resource allocation: The set of chunks that BE users can have access to depends on the allocation of interfering HP users within the cluster. Thus, we denote by $\mathrm{I}_{\mathrm{HP}, \mathrm{BE}}$ the interference matrix of dimensions $|\mathrm{B}| \times \mathrm{H}^{*}$ between $\mathrm{BE}$ and admitted $\mathrm{HP}$ users with 1 or 0 in position $(\mathrm{m}, \mathrm{n})$ according to whether the BE user $\mathrm{m}$ interferes with the HP user $n$ or not (Note that $B$ represents in this case the set of $B E$ users with in the cluster). The resulting matrix $\mathrm{R}_{\mathrm{BE}}=\mathrm{I}_{\mathrm{HP}, \mathrm{BE}}, \times \mathrm{A}_{\mathrm{HP}}$ of dimensions $|\mathrm{B}| \times \mathrm{M}$ can be calculated such that in position $(\mathrm{i}, \mathrm{j}), \mathrm{r}_{\mathrm{ij}}=\sum_{\mathrm{k}=1}^{\left|\mathrm{H}^{*}\right|} \mathrm{I}_{\mathrm{BE}, \mathrm{HP}}(\mathrm{i}, \mathrm{k}) \times \mathrm{A}_{\mathrm{HP}}(\mathrm{k}, \mathrm{j})$. Hence, its complementary matrix $\overline{\mathrm{R}}_{\mathrm{BE}}$ can be defined as:

$$
\overline{\mathrm{r}_{j}}= \begin{cases}1 & \text { If } \mathrm{r}_{\mathrm{ij}}=0 \\ 0 & \text { If } \mathrm{r}_{\mathrm{ij}} \geq 1\end{cases}
$$

Note that $\overline{\mathrm{r}}_{\mathrm{j}}=1$ indicates that the chunk $\mathrm{j}$ can be allocated to the BE user i, hence, the BE users allocation problem can be formulated as follows:

\section{BE users problem:}

- Minimize the maximum value of BE users gaps, i.e., $\min \left(\max _{v \in \mathrm{B}} \mathrm{G}_{\mathrm{BE}}(v)\right]$ 
Subject to:

- BE users should be given chunks less or equal to their demands, i.e., $\forall v \in B: \sum_{j=1}^{\mathrm{M}} \Delta_{\mathrm{BE}}^{\mathrm{v}}(\mathrm{j}) \leq \mathrm{D}_{\mathrm{BE}}^{\mathrm{v}}$

- Two interfering users cannot be given the same chunk, i.e, $\forall \mathrm{j}=1, \ldots, \mathrm{M}, \forall v \in \mathrm{B}, \forall \mathrm{k} \in \mathrm{J}_{\mathrm{v}}: \Delta_{\mathrm{BE}}^{\mathrm{v}}(\mathrm{j})+\Delta_{\mathrm{BE}}^{\mathrm{k}}(\mathrm{j}) \leq 1$

- BE user cannot be given the same chunk as their interfering HP users, i.e, $\forall \mathrm{j}=1, \ldots, \mathrm{M}, \forall v \in \mathrm{B}: \Delta_{\mathrm{BE}}^{\mathrm{v}}(\mathrm{j}) \leq \overline{\mathrm{Y}}_{\mathrm{j}}$

It is worth noting that when assigning chunks inside each cluster, we take into consideration the neighboring clusters. Specifically, for users near the edge of the cluster. Therefore, in the second step, when assigning chunks to the users, each user checks if any of its interfering users, even if they belong to other clusters has the same chunk, the user reports to its FAP about this interference. Hence, the FAP does notallocate this chunk to this user. By doing this, we make sure that after finishing the allocation for all clusters, there will not be a resource contention across clusters.

Performance metrics: The performance of our Enhanced QFCRA (EQFCRA) algorithmis evaluated considering the following QoS metrics. Rate of rejected users, throughput satisfaction rate, spectrum spatial reuse and fairness.

Rate of rejected users: This metric represents the percentage of HP and BE users not admitted into the network. Recall that once accepted, HP users are completely satisfied whereas for BE users their satisfaction degree will be maximized.

Throughput Satisfaction Rate (TSR): TSR denotes the degree of satisfaction of a user with respect to the requested resources. For each user $u$ attached to a FAP $F_{a} \in F, \operatorname{TSR}(u)$ is defined as the ratio of the allocated number of chunks to the requested ones and can be expressed as follows (Hatoum et al., 2013):

$$
\forall \mathrm{u}, \operatorname{TSR}(\mathrm{u})=\sum_{\mathrm{j}=1}^{\mathrm{M}} \Delta^{\mathrm{u}}(\mathrm{j}) / \mathrm{D}^{\mathrm{u}}
$$

For a network with $\mathrm{N}$ users, the TSR metric can be thus given by:

$$
\mathrm{TSR}=\sum_{\mathrm{u}} \mathrm{TSR}(\mathrm{u}) / \mathrm{N}
$$

Spectrum Spatial Reuse (SSR): SSR denotes the average portion of FAPs using the samechunk within the network. Therefore, it is defined as the mean value of chunk's spatial reuse. The SSR metric can be thus, expressed as follows (Hatoum et al., 2013):

$$
\operatorname{SSR}=\frac{1}{\mathrm{M} \times|\mathrm{F}|} \sum_{\mathrm{k}=1}^{\mathrm{M}} \sum_{\mathrm{u} \in \mathrm{H} \cup \mathrm{B}} \Delta^{\mathrm{u}}(\mathrm{k})
$$

Fairness: Fairness is evaluated in terms of the fairness index (Hahne, 1991) which determines how fairly the resources are distributed among $\mathrm{N}$ existing users. It is expressed as follows:

$$
\beta=\left(\sum_{\mathrm{u}=1}^{\mathrm{N}} \operatorname{TSR}(\mathrm{u})\right)^{2} /\left(\mathrm{N} \cdot \sum_{\mathrm{u}=1}^{\mathrm{N}} \operatorname{TSR}(\mathrm{u})^{2}\right)
$$

\section{RESULTS AND DISCUSSION}

In this study, we compare our proposal under various interference scenarios and FAPs densities with QFCRA algorithm proposed by Lee et al. (2010). The number of users in each FAP and their demands vary in each simulation. We consider a typical OFDMA frame (downlink LTE frame) consisting of chunks. Which corresponds to a channel bandwidth of $10 \mathrm{MHz}$ (i.e., 50 chunks in the frequency domain and one sub-frame of $1 \mathrm{msec}$ in length). Scheduling is done on a slot basis. Each sub-frame consists of two equally sized slots of $\mathrm{T}_{\text {slot }}=0.5$ msec, $\mathrm{SC}_{\text {ofdm }}=12$ and $\mathrm{SY}_{\text {ofdm }}=7$ in LTE specification (ETSI TS 136.300 V10.5.0, 2011). Different network sizes are considered (50, 100, 150 and 200) FAPs, varying from low-density networks (50 FAPs) to high-density networks (200 FAPs). The FAPs are distributed randomly over a 2-D 400 $\times 400 \mathrm{~m}$ area with one FAP randomly placed in a $10 \times 10 \mathrm{~m}$ residence. Note that some residences might not have a FAP. We consider each residence is divided into $45 \times 5 \mathrm{~m}$ rooms. Walls between rooms are considered light walls (plastic, wooden,...).

Walls between residences are considered heavy walls (concrete walls). Users are uniformly distributed within the residence area with 10 users max per FAP. These users are divided into $4 \mathrm{HP}$ users with equal priority and $6 \mathrm{BE}$ users. Each user generates its traffic demand which is translated into chunks using (Eq. 1) with a maximum value of 20 chunks per HP user and 10 chunks per BE user. Different minimum required SINR thresholds are considered: 10, 15, 20 and $25 \mathrm{~dB}$ to show the impact of the A1 scenario for indoor small office and residential of Winner for the frequency range 2-6 GHz, each femtocell determines the set of its interfering femtocells depending on the received signal strength from its users. In what follows, we show the results for different SINR thresholds. Our objective is to study how the chunks are effectively assigned to femtocell users considering QoS requirements and interference levels. We focus on rate of rejected users, throughput satisfaction rate, spectrum spatial reuse and fairness among users. The simulation results are obtained using MATLAB. 


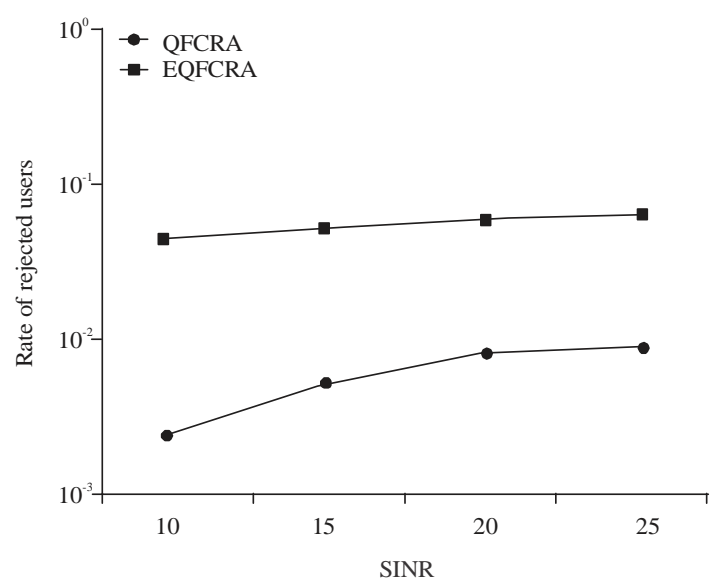

Fig. 1: Rate of rejected users

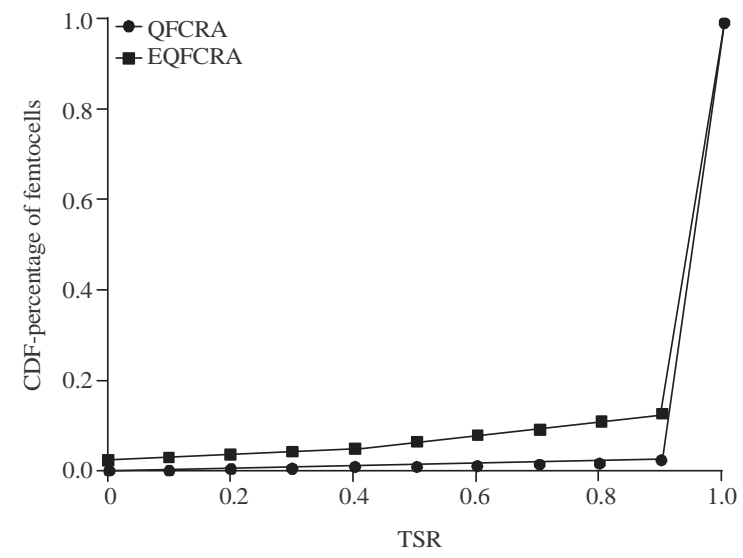

Fig. 2: CDF of throughput satisfaction rate in highdensity networks with low interference level

Rate of rejected users: Figure 1 shows the rate of rejected users for the 200-FAP case. We display the results for all users (HP and $\mathrm{BE}$ ). We can see that EQFCRA allows more than $99.7 \%$ of users to be admitted in the network for low interference levels. Whereas about 99\% for high interference levels, compared with only about 95\% of users for QFCRA in low interference levels. Which degrades to about 93\% for high interference levels.

Throughput Satisfaction Rate (TSR): Figure 2 and 3 show the Cumulative Distributed Function (CDF) of the throughput satisfaction rate for high-density networks in low and high interference levels respectively. We can see that EQFCRA outcomes the QFCRA algorithm in both cases. For QFCRA, about $88 \%$ of the femtocells have their TSR above 0.9. While this number increase to about 97\% for EQFCRA. This performance degrades for high interference levels where it reaches $81 \%$ for QFCRA and 95\% for EQFCRA. This is because in QFCRA there is a stage to resolve the inter-cluster resource contention. Where some of the users lose one or more chunk of their allocated ones which affects the performance.

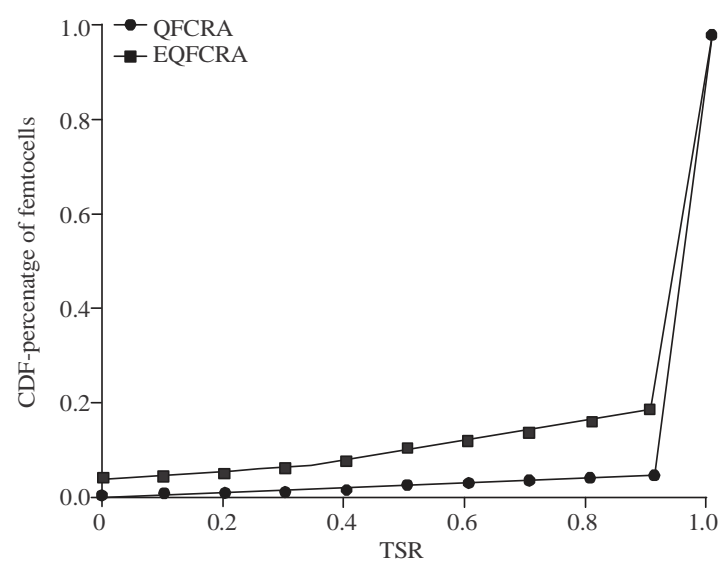

Fig. 3: CDF of throughput satisfaction rate in high-density networks with high interference level

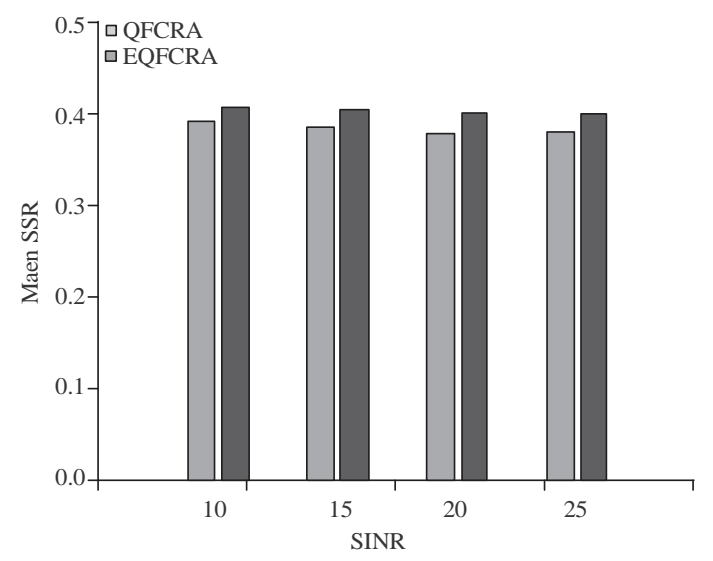

Fig. 4: Mean SSR vs. SINR

Spectrum Spatial Reuse (SSR): Figure 4 plots the mean SSR as function of SINR for high-density networks. We can observe two things. First, EQFCRA offer higher SSR values than QFCRA for all interference levels. Where the gain can attain $2 \%$. Second, we can notice that the SSR metric decreases with the increase of SINR for both strategies, since, the interference degree of each FAP increases.

Fairness: It is important to assess if the resources are fairly distributed between users. Figure 5 shows the fairness metric calculated as the average for the entire network. In the best case, it is equal to 1 when all users receive resources as much as they demand. We can see for high-density network that fairness for QFCRA vary from 95\% to about $92.5 \%$ for low and high interference levels, respectively. While for EQFCRA, it ranges from about $99.5-98 \%$ for low and high interference levels, respectively. This improvement is due to the optimization phase of allocation per cluster. 


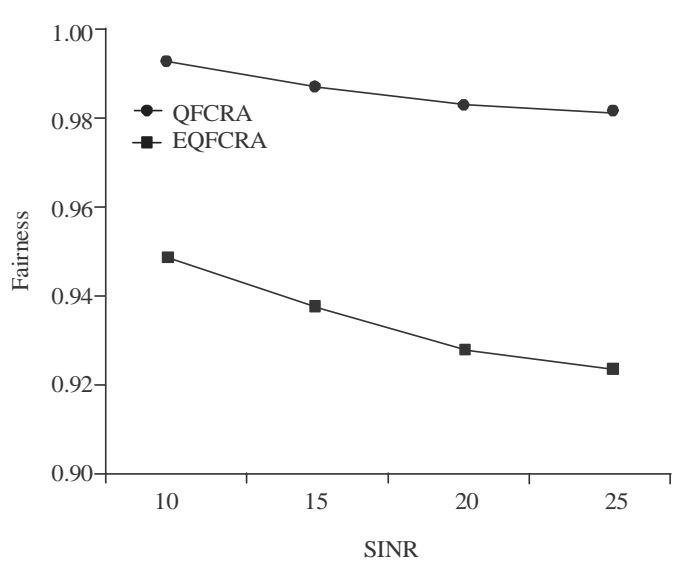

Fig. 5: Fairness

\section{CONCLUSION}

In this study, we studied the joint resource allocation and admission control problem in downlink OFDMA clustered-femtocell networks. Our objective was to study how the chunks are effectively assigned to femtocell users considering QoS requirements and interference levels for high density networks. Two types of users are considered: HP users and BE users. HP users are strict with their demands and must be satisfied. BE users are served effectively as possible. Resolving the allocation problem consists of two stages: cluster formation, resource allocation per cluster. We took into account neighboring clusters when resolving the allocation problem for each cluster so that a user is not given a resource if any of their interferers has the chunk. By doing so, we did not need to worry about contention between neighboring clusters and this improved the performance as we saw in the results section. We used the QFCRA algorithm as a benchmark to compare the performance with it. Through dedicated simulations, we showed that our proposal could achieve significant gains in terms of rate of rejected users throughput satisfaction rate, spectrum spatial reuse and fairness among users, compared to QFCRA. Which lead to expand the network capacity. It is worth noting that the algorithm design is based on OFDMA networks, so, the principle and the algorithmic essence are also applicable to other OFDMA-based wireless networks. Finally, results proved that our proposal outperforms an existing approach, QFCRA for all considered metrics. Which makesit an efficient solution for resource allocation in high density femtocell networks.

\section{REFERENCES}

Aissi, H., C. Bazgan and D. Vanderpooten, 2005. Complexity of the min-max and min-max regret assignment problems. Oper. Res. Lett., 33: 634-640.
Bouras, C. and G. Diles, 2015. Resource management in $5 \mathrm{G}$ femtocell networks. Proceedings of the 2015 10th International Conference on Broadband and Wireless Computing, Communication and Applications (BWCCA'2015), November 4-6, 2015, IEEE, Krakow, Poland, pp: 353-358.

Chandrasekhar, V. and J.G. Andrews, 2009. Spectrum allocation in tiered cellular networks. IEEE Trans. Commun., 57: 3059-3068.

Chinneck, J.W. and E.W. Dravnieks, 1991. Locating minimal infeasible constraint sets in linear programs. Informs J. Comput., 3: 157-168.

Day, R. and P. Milgrom, 2008. Core-selecting package auctions. Int. J. Game Theory, 36: 393-407.

ETSI TS 136.300 V10.5.0, 2011. LTE: Evolved universal terrestrial radio access (E-UTRA) and evolved universal terrestrial radio access network (E-UTRAN). 3GPP, France, Europe. https://www.etsi.org/ deliver/etsi_ts/136300_136399/136300/10.05.00_60 /ts_136300v100500p.pdf

Ghaith, A., R. Hatoum and A. Hatoum, 2016. Joint resource allocation algorithms for downlink in LTE and 5 G networks. Eur. Int. J. Sci. Technol., 5: 16-30.

Hahne, E.L., 1991. Round-robin scheduling for max-min fairness in data networks. IEEE. J. Sel. Areas Commun., 9: 1024-1039.

Hatoum, A., R. Langar, N. Aitsaadi, R. Boutaba and G. Pujolle, 2013. Cluster-based resource management in OFDMA femtocell networks with QoS guarantees. IEEE. Trans. Veh. Technol., 63: 2378-2391.

Jin, M.S., S.A. Chae and D.I. Kim, 2011. Per cluster based opportunistic power control for heterogeneous networks. Proceedings of the 2011 IEEE 73rd Vehicular Technology Conference (VTC Spring'11), May 15-18, 2011, IEEE, Yokohama, Japan, pp: 1-5.

Lee, H.C., D.C. Oh and Y.H. Lee, 2010. Mitigation of inter-femtocell interference with adaptive fractional frequency reuse. Proceedings of the 2010 IEEE International Conference on Communications, May 23-27, 2010, IEEE, Cape Town, South Africa, pp: 1-5.

Lee, J. and M. Lee, 2015. Resource allocation with QoS supporting in macro-femtocell networks. Int. J. Multimedia Ubiquitous Eng., 10: 365-378.

Lee, T., H. Kim, J. Park and J. Shin, 2010. An efficient resource allocation in OFDMA femtocells networks. Proceedings of the 2010 IEEE 72nd International Conference on Vehicular Technology Fall (VTC-Fall'10), September 6-9, 2010, IEEE, Ottawa, Canada, pp: 1-5.

Li, L., C. Xu and M. Tao, 2012. Resource allocation in open access OFDMA femtocell networks. IEEE. Wirel. Commun. Lett., 1: 625-628. 
Li, Y.Y. and E.S. Sousa, 2012. Cognitive femtocell: A cost-effective approach towards $4 \mathrm{G}$ autonomous infrastructure networks. Wireless Pers. Commun., 64: 65-78.

Liang, Y.S., W.H. Chung, G.K. Ni, I.Y. Chen, H. Zhang and S.Y. Kuo, 2012. Resource allocation with interference avoidance in OFDMA femtocell networks. IEEE. Trans. Veh. Technol., 61: 2243-2255.

Lopez-Perez, D., A. Valcarce, G. de la Roche and J. Zhang, 2009. OFDMA femtocells: A roadmap on interference avoidance. IEEE. Commun. Mag., 47: 41-48.

Mohammadi, A., M.J. Dehghani and E. Ghazizadeh, 2018. Game theoretic spectrum allocation in femtocell networks for smart electric distribution grids. Energies, Vol. 11, 10.3390/en11071635

Pourkabirian, A., M.D.T. Fooladi, E. Zeinali and A.M. Rahmani, 2018. Dynamic resource allocation for OFDMA femtocell networks: A game-theoretic approach. Telecommun. Syst., 69: 51-59.
Sahin, M.E., I. Guvenc, M.R. Jeong and H. Arslan, 2009. Handling CCI and ICI in OFDMA femtocell networks through frequency scheduling. IEEE Trans. Consum. Electron., 55: 1936-1944.

Sundaresan, K. and S. Rangarajan, 2009. Efficient resource management in OFDMA femto cells. Proceedings of the 10th ACM International Symposium on Mobile Ad Hoc Networking and Computing, May 18-21, 2009, Louisiana, USA., pp: 33-42.

TS 38.211, NR, 2019. Physical channels and modulation. 3GPP, France, Europe.

Vaezpour, E., M. Dehghan and H. Yousefi'zadeh, 2017. Robust distributed resource allocation in OFDMA femtocell networks. Comput. Commun., 109: 1-12.

Zhang, H., C. Jiang, N.C. Beaulieu, X. Chu, X. Wen and M. Tao, 2014. Resource allocation in spectrum-sharing OFDMA femtocells with heterogeneous services. IEE. Tran. Commun., 62: 2366-2377. 